\title{
The experience of contact tracing in Singapore in the control of COVID-19: highlighting the use of digital technology
}

\author{
Sean Han Sheng Lai ${ }^{1} \cdot$ Camelia Qian Ying Tang ${ }^{2} \cdot$ Asok Kurup $^{3} \cdot$ Gowreeson Thevendran $^{4}$ (D)
}

Received: 13 May 2020 / Accepted: 18 May 2020 / Published online: 14 November 2020

(C) SICOT aisbl 2020

\begin{abstract}
Purpose Containing the coronavirus disease 2019 (COVID-19) pandemic would require aggressive contact tracing and isolation of suspected or confirmed COVID-19 cases. Models in published literature have suggested that digital rather than manual contact tracing might be more effective in containing the pandemic. This article seeks to examine the forms of contact tracing that Singapore, a highly dense city-state, adopts with a focus on new innovations including the use of digital technology.

Methods An exploratory literature search in PubMed, MEDLINE and EMBASE for studies reviewing technological approaches and responses to COVID-19 was performed. Information published by national agencies was also analysed to ascertain how technology was utilised in contact tracing.

Results Contact tracing in Singapore is overseen by the Ministry of Health (MOH). COVID-19 cases are interviewed on their whereabouts during a backward and forward activity mapping process to identify close contacts. Extensive contact tracing even involving the police and serological tools have helped to establish links between cases and closed several local clusters. Examination of patient's digital footprint has helped in contact tracing. Other digital technology introduced includes SafeEntry and TraceTogether. SafeEntry is a cloud-based visitor registration system while TraceTogether is a mobile phone application which operates by exchanging anonymised identifiers between nearby phones via Bluetooth connection.

Conclusion Digital contact tracing is likely to expand and continue to complement human-based contact tracing for the current and future pandemics. However, at this juncture, it is not ready to replace the manual and meticulous work that only Singapore contact tracers can achieve.
\end{abstract}

Keywords Coronavirus $\cdot$ COVID-19 $\cdot$ Digital contact tracing $\cdot$ Contact tracing

\section{Introduction}

The coronavirus disease 2019 (COVID-19) is caused by the virus severe acute respiratory syndrome coronavirus 2 (SARS-CoV-2) [1]. First detected in Wuhan, China, in December 2019, it quickly evolved into a global pandemic,

Gowreeson Thevendran

xanthus23@hotmail.com

1 Yong Loo Lin School of Medicine, National University of Singapore, Singapore, Singapore

2 Department of Hand and Reconstructive Microsurgery, Tan Tock Seng Hospital, Tan Tock Seng, Singapore

3 Mount Elizabeth Medical Centre, \#03-01, Mount Elizabeth, Singapore

4 Consultant Orthopaedic Surgeon, Mount Elizabeth Novena Hospital, Singapore, Singapore culminating in the World Health Organization (WHO) declaring a state of global pandemic on the 11th of March 2020. At the time of writing, there has been more than three million confirmed cases of COVID-19 and more than 200,000 deaths globally [2]. In the absence of mitigation, it is estimated that COVID-19 could result in seven billion infections and 40 million deaths worldwide this year [3].

Viral pandemic containment strategies can be broadly classified into antivirals, vaccines and non-pharmacological measures. Globally, responses need to be swift to curtail the spread of the pandemic and this would involve aggressive surveillance, contact tracing, isolating and quarantining suspected and confirmed COVID-19 cases. Models published in existing literature have suggested that the practice of manual contact tracing faces challenges in limiting transmission as it is too slow and cannot be scaled up once the epidemic grows beyond the early phases due to limited manpower [4-6]. It has also been suggested that a digital form of contact tracing might be more 
effective [7]. The purpose of this article is to examine the forms of contact tracing that Singapore adopts in an effort to contain the pandemic in a highly dense city-state with a focus on new innovations including the use of digital technology.

\section{Material and methods}

An exploratory literature search for technological approaches and responses to COVID-19 was performed. A search of published literature in PubMed, MEDLINE and EMBASE was undertaken to include publications published between the 31st of December 2019 and 6th of May 2020. Search terms used included "coronavirus", "COVID-19", " contact tracing" and "digital contact tracing". There was a paucity of publications on whole-of-government efforts in contact tracing and use of digital technology to support this endeavour.

Information published by the various national agencies were analysed to ascertain how technology was utilised in contact tracing. These measures were also reviewed with regard to their position within existing regulatory and legal framework. Shortcomings were identified and possible rectifying measures reviewed. These disadvantages were weighed against potential benefits to assess the relevance of these measures.

\section{Results}

Contact tracing in Singapore is overseen by the Ministry of Health $(\mathrm{MOH})$ and involves many other stakeholders including local hospitals, paramedics from the Singapore Civil Defence Force (SCDF), police officers from the Singapore Police Force (SPF), Certis security officers, the Government Technology Agency (GovTech) and volunteers from the various statutory boards. This team rapidly swung into action ever since the first case of Covid-19 was detected in Singapore on the 23rd of January 2020 [8]. As part of the contact tracing process, COVID-19 cases were interviewed on their whereabouts during a backward and forward activity mapping process to identify close contacts who can then be quarantined or monitored closely. The data is used by epidemiologists to inform public health strategies.

At the work front, while telecommuting is encouraged, all workplaces in Singapore are required by law to have a system in place to log employees' entry and exit from their workplaces for contact tracing purposes. Besides that, they are also required to implement measures to avoid transmission of COVID-19 at and across workplace premises. This includes prohibiting teams working in different locations to physically interact with one another, ensuring employees wear masks at their workplaces and implementing safe distancing measures [9]. In addition to traditional epidemiological methods of contact tracing, Singapore has also been credited as a world-first in innovating and utilising a SARS-CoV-2 serological analysis tool to establish the link between 2 large clusters of COVID-19 cases and subsequently tracing the transmission chain to an imported source $[10,11]$. To complement contact tracing, border control measures such as travel restrictions and mandatory 14 days isolation of travellers at designated facilities help to ring-fence the risk of community transmission of the disease from imported cases.

Nonetheless, contact tracing is expectedly time consuming, laborious and with possible recall bias, especially if patients are unwell. Digital technology has come to aid in several ways. One technique that the authorities in Singapore are using is the examination of patient's digital footprint [12]. ATM withdrawals and credit card activities, such as through ridesharing applications, credit card payments at restaurants or shopping centres, movement on public transportation, leave digital footprints and these can assist the authorities in finding out where the person has been and how they have travelled. $\mathrm{MOH}$ also maintains a live COVID-19 dashboard on the ministry's webpage providing latest updates on the situation in the country [13]. This website has been commended by other countries and Singapore's contact tracing efforts have been complimented as one of the best in the world [12]. Apart from receiving updates from the website, Singaporeans can also subscribe to WhatsApp and Telegram updates from the ministry as well.

Another digital technology that Singapore has utilised is SafeEntry, which is a national free-for-use cloud-based visitor registration system [14]. SafeEntry logs visits by individuals to hotspots and venues providing essential services. Individuals visiting these premises are required to provide key personal information such as name, National Registration Identity Card (NRIC) number and mobile phone number. Premises would display the SafeEntry Quick Response (QR) code poster prominently, and individuals would scan the designated QR code using their phones to access the SafeEntry website where they can consent to the sharing of their personal details via the SingPass Mobile application which allows Singaporeans to access government e-services, or key it in manually if they do not have a SingPass Mobile account. When individuals leave the premise, they need to log their check out time with the system. The SafeEntry service was met with good response and has been utilised by businesses at over 40,000 locations. Collected data is encrypted, and the database can only be accessed by authorised public officers for contact tracing purposes. The data is purged when it is no longer needed for contact tracing [15].

Another digital contact tracing technology that has been introduced by the Singapore government is the TraceTogether mobile phone application [16]. TraceTogether operates by exchanging anonymised identifiers between nearby phones via a Bluetooth connection. During the exchange, only four pieces of information are collected: a timestamp, Bluetooth signal strength, the phone's model and a temporary identifier or device 
nickname. This information is stored locally on the user's phone in an encrypted form. If a user of TraceTogether application is diagnosed with COVID-19, the user will be asked to give contact tracers access to the TraceTogether data which is the list of anonymised identifiers that the user's phone has been close to. This information will then be decrypted, thereby allowing identification of other TraceTogether users who have been in close contact with the user. Developers around the world can now access the building blocks of Singapore's contact tracing application TraceTogether for free and make their own version to turn the tide against Covid-19 [17]. The application's code is now officially open-sourced and developers can find out more about TraceTogether's source code, the BlueTrace protocol, at bluetrace.io.

At the time of writing, more countries are adopting similar digital contact tracing measures. In the USA, initial efforts of contact tracing involved scaling up contact tracing workforce [18], which ultimately kick-started various digital contact tracing initiatives [19]. Technologies that have been introduced include Covid Watch from Stanford University [20] and CoEpi [21] which are anonymous Bluetooth proximitybased exposure alerting systems, as well as SafePaths developed by a team from the Massachusetts Institute of Technology (MIT) which utilised an anonymised 28-day Global Positioning System (GPS) trajectory [22]. Such efforts are also apparent in Europe, with efforts underway to develop General Data Protection Regulation (GDPR)-compliant platform to enable anonymous contact tracing as part of the PanEuropean Privacy-Preserving Proximity Tracing (PEPP-PT) [23] project, though this technology is not operational in Europe yet at the time of writing. The Australian government has launched a Bluetooth proximity-based application called COVIDSafe for contact tracing purposes [24], while New Zealand has also announced similar plans for the future [25].

In China, the "Close contact detector" geosocial application [26] primarily makes use of scanning of QR codes via popular mobile social and payment applications to record the locations its citizens have been. This is in contrast to South Korea, where a multiprong approach involving the use of GPS, card transactions, surveillance cameras and cell phone data was utilized in contact tracing [27].

\section{Discussion}

The experience of contact tracing in Singapore was built up over years of preparedness after previous experiences of pandemics such as Severe Acute Respiratory Syndrome (SARS) pandemic in 2003 and H1N1 Influenza pandemic in 2009. From the outset, Singapore's strategy of using a comprehensive surveillance system to detect as many cases as possible and to contain them at the individual level was effective. Despite early importations resulting in local chains of transmission, the rise in the number of cases has been steady without the exponential growth observed elsewhere. A study by Harvard University's Center for Communicable Disease Dynamics estimates that Singapore detects nearly three times the number of imported cases than the global average due to its strong epidemiological surveillance and contact tracing processes [28]. Limited understanding of the disease in the early period of the outbreak led to quarantining of exposed individuals only from the day of illness in the index. It became clear later that although the median incubation period is approximately 4 to 5 days, with $97.5 \%$ of patients having symptoms within 11.5 days of exposure [29], there was a presymptomatic phase of active transmission [30-32]. In an analysis of 157 locally acquired COVID-19 cases in Singapore, $6.4 \%$ of these cases were attributed to pre-symptomatic transmission, with the exposure occurring 1 to 3 days before the source patient developed symptoms [33]. A modelling study by Yuan et al. that evaluated the effectiveness of quarantine measures on the transmissibility of COVID-19 in Hong Kong noted the timing of quarantine to be a major determinant in the risk of community transmission [34]. According to the study, a quarantine time of half a day before symptom onset is able to reduce the basic reproduction number (R0) from 2.32 to 0.76 , whereas a quarantine time of more than a day after symptom onset led to an R0 of more than 1. In addition, timing to quarantine is also paramount in determining detection efficiency, with a 1-day delay and 6-day delay in quarantine reducing the daily detection ratio from 71 to $60 \%$ and $31 \%$ respectively.

Local policies have since changed to include the presymptomatic phase in contact mapping and quarantining efforts. However, it was the very extensive and arduous work undertaken by contact tracers and public health experts which led to the studies underscoring the relevance of pre-symptomatic transmission which in turn led to changes in policies. Extensive contact tracing even with the help of the police and serological tools have helped to establish links between cases and close several local clusters. Even now, new clusters are constantly being detected because of this process. As of now, the number of cases in the community have reduced from an average of 41 new cases daily (week of 7th April to 13th April) to 8 new cases daily (week of 5th of May to 11th of May) [35]. This is the result of multi-pronged interventions including enforced social distancing, masking in public, closure of nonessential services and businesses, complemented by aggressive testing strategies, with contact tracing and isolation.

Digital contact tracing is not a new initiative. The use of mobile devices for contact tracing has long been recognised, with various strategies and models developed to utilise communication traces by mobile phones for proxies of physical interactions [36]. The idea of smartphone application for contact tracing was used in Sierra Leone during the 2014-2016 Ebola epidemic and demonstrated the potential to improve surveillance data via digital applications to collect and analyse contact tracing data [37]. In Botswana, a study by Yoonhee et al. evaluated the use of a mobile phone application over a 6- 
month period in tuberculosis (TB) contact tracing and found that it reduced the time required to complete TB contact tracing per contact and improved quality of data collected compared to paper form-based approach which required writing, manual entry of data into a database and manual generation of summary reports [38].

The effectiveness of any contact tracing system depends on the level of public support. As of the 1st of April 2020, a million people in Singapore have the contact-tracing application TraceTogether, but at least three-quarters of the population (approximately four million) needs to have it for the application to be effective [39]. A significant concern is the encroachment of data privacy and the possibility that the application could be used as a means of greater surveillance by the government. The governing body would also have to show that they would be able to defend against security risk posed by malicious hackers who would like to obtain these data. Lastly, crime prevention agencies would have to deal with phishing by criminals pretending to be contact tracers $[40,41]$.

It is crucial to find a balance between deployment of the technology and safeguarding data safety and patient privacy. The Singapore government has reassured its population that the application TraceTogether does not track location or contacts, and data is stored locally on the phone for 21 days and will not be accessed unless the individual has been identified as a close contact, and measures are in place to protect the individual's mobile number [42]. Nonetheless, despite improvements in contact tracing technology, Singapore's contact tracers will likely remain at the frontline of the fight against COVID-19 because only they can discriminate between multiple sources of data and add a human touch in interviews with people exposed to SARS-CoV-2.

In conclusion, digital contact tracing is likely to expand and continue to complement human-based contact tracing for the current and future pandemics. However, it is not ready to replace the manual and meticulous work that only Singapore contact tracers can achieve.

\section{Compliance with ethical standards}

Conflict of interests The authors declare that they have no conflicts of interest.

\section{References}

1. World Health Organization (2020) Naming the Coronavirus disease (COVID-19) and the Virus that Causes It. World Health Organization. https://www.who.int/emergencies/diseases/novelcoronavirus-2019/technical-guidance/naming-the-coronavirusdisease-(covid-2019)-and-the-virus-that-causes-it. Accessed 30th April 2020

2. World Health Organization (2020) Coronavirus disease (COVID2019) situation reports. World Health Organization. https://www. who.int/emergencies/diseases/novel-coronavirus-2019/situationreports. Accessed 30th April 2020

3. Walker PG, Whittaker C, Watson O, Baguelin M, Ainslie KEC, Bhatia S, Bhatt S, Boonyasiri A, Boyd O, Cattarino L, Cucunubá Z, Cuomo-Dannenburg G, Dighe A, Donnelly CA, Dorigatti I, Elsland Sv, FitzJohn R, Flaxman S, Fu H, Gaythorpe K, Geidelberg L, Grassly N, Green W, Hamlet A, Hauck K, Haw D, Hayes S, Hinsley W, Imai N, Jorgensen D, Knock E, Laydon D, Mishra S, Nedjati-Gilani G, Okell LC, Riley S, Thompson H, Unwin J, Verity R, Vollmer M, Walters C, Wang HW, Wang Y, Winskill P, Xi X, Ferguson NM, Ghani AC (2020) The global impact of COVID-19 and strategies for mitigation and suppression. Imperial College London doi:https://doi.org/10.25561/77735

4. Klinkenberg D, Fraser C, Heesterbeek H (2006) The effectiveness of contact tracing in emerging epidemics. PLoS One 1:e12. https:// doi.org/10.1371/journal.pone.0000012

5. Peak CM, Childs LM, Grad YH, Buckee CO (2017) Comparing nonpharmaceutical interventions for containing emerging epidemics. Proc Natl Acad Sci U S A 114(15):4023-4028. https:// doi.org/10.1073/pnas.1616438114

6. Hellewell J, Abbott S, Gimma A, Bosse NI, Jarvis CI, Russell TW, Munday JD, Kucharski AJ, Edmunds WJ, Centre for the Mathematical Modelling of Infectious Diseases C-WG, Funk S, Eggo RM (2020) Feasibility of controlling COVID-19 outbreaks by isolation of cases and contacts. Lancet Glob Health 8(4):e488e496. https://doi.org/10.1016/S2214-109X(20)30074-7

7. Ferretti L, Wymant C, Kendall M, Zhao L, Nurtay A, AbelerDörner L, Parker M, Bonsall D, Fraser C (2020) Quantifying SARS-CoV-2 transmission suggests epidemic control with digital contact tracing. Science:eabb6936. https://doi.org/10.1126/science. abb6936

8. Abdullah Z, Salamat H (2020) Singapore confirms first case of Wuhan virus. Channel NewsAsia. https://www.channelnewsasia. com/news/singapore/wuhan-virus-pneumonia-singapore-confirmsfirst-case-12312860. Accessed 4th May 2020

9. Ministry of Health Singapore (2020) Strong national push to stem spread of COVID-19. Government of Singapore. https://www. moh.gov.sg/news-highlights/details/strong-national-push-to-stemspread-of-covid-19. Accessed 1 May 2020

10. Yong SEF, Anderson DE, Wei WE, Pang J, Chia WN, Tan CW, Teoh YL, Rajendram P, Toh M, Poh C, Koh VTJ, Lum J, Suhaimi NM, Chia PY, Chen MI, Vasoo S, Ong B, Leo YS, Wang L, Lee VJM (2020) Connecting clusters of COVID-19: an epidemiological and serological investigation. Lancet Infect Dis. https://doi.org/10. 1016/s1473-3099(20)30273-5

11. CNA (2020) Duke-NUS used COVID-19 antibody tests to establish link between church clusters in a world-first. Mediacorp. https://www.channelnewsasia.com/news/singapore/covid19coronavirus-duke-nus-antibody-tests-12469184. Accessed 5th May 2020

12. Sagar M (2020) The importance of contact tracing in Singapore and the role technology plays. OpenGov Asia. https://www. opengovasia.com/the-importance-of-contact-tracing-in-singaporeand-the-role-technology-plays/. Accessed 5th May 2020

13. Ministry of Health Singapore (2020) Updates on COVID-19 (coronavirus disease 2019) local situation. Government of Singapore. https://www.moh.gov.sg/covid-19. Accessed 5th May 2020

14. Government Technology Agency (2020) SafeEntry. Government of Singapore. https://www.ndi-api.gov.sg/safeentry. Accessed 13 May 2020

15. SafeEntry (2020) How will my data be protected? Government of Singapore. https://support.safeentry.gov.sg/hc/en-us/articles/ 900000681226\%2D\%2DHow-will-my-data-be-protected-. Accessed 1 May 2020

16. NDI Developer and Partner Portal (2020) TraceTogether - behind the scenes look at its development process. Government 
Technology Agency. https://www.tech.gov.sg/media/technews/ tracetogether-behind-the-scenes-look-at-its-development-process. Accessed 1 May 2020

17. Sharwood S (2020) Singapore to open-source national coronavirus encounter-tracing app and the Bluetooth research behind it. Situation Publishing. https://www.theregister.co.uk/2020/03/26/ singapore_tracetogether_coronavirus_encounter_tracing_app_ lessons/. Accessed 6 May 2020

18. Watson C, Cicero A, Blumenstock J, Fraser M (2020) A national plan to enable comprehensive COVID-19 case finding and contact tracing in the US. Johns Hopkins University. https://www. centerforhealthsecurity.org/our-work/pubs_archive/pubs-pdfs/ 2020/200410-national-plan-to-contact-tracing.pdf. Accessed 1 May 2020

19. Digital Contact Tracing Tools for COVID-19 (2020). https://www. cdc.gov/coronavirus/2019-ncov/downloads/digital-contact-tracing. pdf. Accessed 1 May 2020

20. Covid Watch (2020). https://www.covid-watch.org/about. Accessed 1st May 2020

21. About CoEpi. https://www.coepi.org/about/. Accessed 1 May 2020

22. Project Overview « Safe Paths - MIT Media Lab. (2020) MIT Media Lab. https://www.media.mit.edu/projects/safepaths/ overview/. Accessed 1 May 2020

23. Pan-European Privacy-Preserving Proximity Tracing (PEPP-PT). Pepp-pt.org. https://www.pepp-pt.org/. Accessed 1 May 2020

24. Taylor J (2020) Covidsafe app: how to download Australia's coronavirus contact tracing app, how it works, What It Does and Problems. The Guardian. https://www.theguardian.com/australianews/2020/may/05/covid-safe-app-downloads-ios-android-iphoneaustralian-government-covidsafe-tracking-how-to-downloadinstall-works-working-problems-australia-coronavirus-contacttracing. Accessed 6 May 2020

25. Walls J (2020) Covid 19 coronavirus: NZ's own tracing app on way as Australia rolls out COVIDSafe. New Zealand Herald. https:// www.nzherald.co.nz/nz/news/article.cfm?c_id=1\&objectid= 12327738. Accessed 6 May 2020

26. Kamel Boulos MN, Geraghty EM (2020) Geographical tracking and mapping of coronavirus disease COVID-19/severe acute respiratory syndrome coronavirus 2 (SARS-CoV-2) epidemic and associated events around the world: how 21st century GIS technologies are supporting the global fight against outbreaks and epidemics. Int J Health Geogr 19(1):8. https://doi.org/10.1186/s12942-02000202-8

27. Covid-19 National Emergency Response Center, Epidemiology \& Case Management Team, Korea Centers for Disease Control \& Prevention (2020) Contact Transmission of COVID-19 in South Korea: Novel Investigation Techniques for Tracing Contacts. Osong Public Health Res Perspect 11(1):60-63. https://doi.org/10. 24171/j.phrp.2020.11.1.09

28. Niehus R, De Salazar PM, Taylor A, Lipsitch M (2020) Quantifying bias of COVID-19 prevalence and severity estimates in Wuhan, China that depend on reported cases in international travelers. medRxiv:2020.2002.2013.20022707. doi:https://doi. org/10.1101/2020.02.13.20022707

29. Lauer SA, Grantz KH, Bi Q, Jones FK, Zheng Q, Meredith HR, Azman AS, Reich NG, Lessler J (2020) The incubation period of coronavirus disease 2019 (COVID-19) from publicly reported confirmed cases: estimation and application. Ann Intern Med. https:// doi.org/10.7326/M20-0504

30. Rothe C, Schunk M, Sothmann P, Bretzel G, Froeschl G, Wallrauch C, Zimmer T, Thiel V, Janke C, Guggemos W, Seilmaier M,
Drosten C, Vollmar P, Zwirglmaier K, Zange S, Wolfel R, Hoelscher M (2020) Transmission of 2019-nCoV infection from an asymptomatic contact in Germany. N Engl J Med 382(10): 970-971. https://doi.org/10.1056/NEJMc2001468

31. Yu P, Zhu J, Zhang Z, Han Y, Huang L (2020) A familial cluster of infection associated with the 2019 novel coronavirus indicating potential person-to-person transmission during the incubation period. J Infect Dis. https://doi.org/10.1093/infdis/jiaa077

32. Bai Y, Yao L, Wei T, Tian F, Jin DY, Chen L, Wang M (2020) Presumed asymptomatic carrier transmission of COVID-19. JAMA. https://doi.org/10.1001/jama.2020.2565

33. Wei WE, Li Z, Chiew CJ, Yong SE, Toh MP, Lee VJ (2020) Presymptomatic transmission of SARS-CoV-2 - Singapore, January 23-March 16, 2020. MMWR Morb Mortal Wkly Rep 69(14):411-415. https://doi.org/10.15585/mmwr.mm6914e1

34. Yuan H-Y, Mao A, Han G, Yuan H, Pfeiffer D (2020) Effectiveness of quarantine measure on transmission dynamics of COVID-19 in Hong Kong. medRxiv:2020.2004.2009.20059006. doi:https://doi.org/10.1101/2020.04.09.20059006

35. Lai L (2020) S'pore's Covid-19 circuit breaker measures have shown promise, could be further eased on June 1. The Straits Times. https://www.straitstimes.com/singapore/circuit-breakermeasures-have-shown-promise-could-be-further-eased-on-june-1. Accessed 13 May 2020

36. Farrahi K, Emonet R, Cebrian M (2014) Epidemic contact tracing via communication traces. PLoS One 9(5):e95133. https://doi.org/ 10.1371/journal.pone.0095133

37. Danquah LO, Hasham N, MacFarlane M, Conteh FE, Momoh F, Tedesco AA, Jambai A, Ross DA, Weiss HA (2019) Use of a mobile application for Ebola contact tracing and monitoring in northern Sierra Leone: a proof-of-concept study. BMC Infect Dis 19(1):810. https://doi.org/10.1186/s12879-019-4354-z

38. Ha YP, Tesfalul MA, Littman-Quinn R, Antwi C, Green RS, Mapila TO, Bellamy SL, Ncube RT, Mugisha K, Ho-Foster AR, Luberti AA, Holmes JH, Steenhoff AP, Kovarik CL (2016) Evaluation of a mobile health approach to tuberculosis contact tracing in Botswana. J Health Commun 21(10):1115-1121. https://doi. org/10.1080/10810730.2016.1222035

39. Chong C (2020) About 1 million people have downloaded TraceTogether app, but more need to do so for it to be effective: Lawrence Wong. The Straits Times. https://www.straitstimes.com/ singapore/about-one-million-people-have-downloaded-thetracetogether-app-but-more-need-to-do-so-for. Accessed 1st May 2020

40. Reuters (2020) True claim: COVID-19 proximity text messages are fake. Reuters. https://www.reuters.com/article/uk-factcheckcoronavirus-proximity-text/true-claim-covid-19-proximity-textmessages-are-fake-idUSKBN222205. Accessed 1st May 2020

41. Kaur H, Lee A (2020) Beware of these fake text messages and Robocalls going around about the coronavirus. Cable News Network. https://edition.cnn.com/2020/04/19/us/coronavirus-textmessage-scam-trnd/index.html. Accessed 1st May 2020

42. Ministry of Communications and Information (2020) Help speed up contact tracing with TraceTogether. Government of Singapore. https://www.gov.sg/article/help-speed-up-contact-tracing-withtracetogether. Accessed 1st May 2020

Publisher's note Springer Nature remains neutral with regard to jurisdictional claims in published maps and institutional affiliations. 